\title{
Peer Reviewers
}

\begin{tabular}{ll} 
Mariel Aguilar-Stoen & Orou Gaoue (3) \\
Jose A. Alayon-Gamboa & Peter Giovannini \\
Ulysses Paulino Albuquerque (2) & Ana María Gómez Caravaca \\
Miguel Alexiades & Bryan Hanson (3) \\
Achille Assogbadjo & Karen Hardy \\
Dan Austin (2) & Robbie Hart (2) \\
Henrik Balslev & Franz Huber \\
Brad Bennett & Michael J. Balick \\
Stephen Brush (2) & Katie Konchar \\
Rainer W Bussmann (2) & Donovan Kotze \\
Laura Calvet-Mir & Vijesh Krishna \\
Laura Calvet-Mir & Monika Kujawska \\
Rodrigo Camara-Leret (2) & David Lentz \\
Dominique Cardon & T. Abe Lloyd \\
Mary Carrington & Chunlin Long \\
Alejandro Casas & Lukasz Luczaj \\
Charles R. Clement & Manuel Macia \\
Felix Coe & Laurent Marquer \\
Oliver Coomes (2) & Susanne Masters \\
Alain Cuerrier & Patricia Muniz Medeiros \\
Ugo D'Ambrosio & Felipe Melo \\
Hugo de Boer & Daniel E. Moerman (4) \\
Fernanda de Costa & Maria Molina \\
Jillian De Gezelle & Mónica Moraes \\
John de la Parra & Christopher Morehart \\
Reinaldo Farias Paiva de & Kathy Parker \\
Lucena & Gregory Plunkett \\
Maurício Sedrez Dos Reis & Zbynek Polesny \\
Eve Emshwiller (3) & Cassandra Quave \\
Abdeslam Ennabili & Cassandra Quave \\
Washington Ferreira Junior & John Rashford \\
\hline &
\end{tabular}

Victoria Reyes-Garcia (3)

Janet Rock

Katia Angélica Figueroa

Rodríguez

Nanci Ross (4)

Anirban Roy

John Paul Schmit

Mauro Serafini

Charlie Shackleton

Pat Shipman

Rafael Silva

Ranjay K. Singh

Joanna Sosnowska

John Richard Stepp (2)

Tamara Ticktin

Alexandra Towns

Virgilio Uarrota

Tinde van Andel

Ina Vandebroek (3)

Alejandra Vilela (2)

Christian Reinhard Vogl (2)

Valeria Volin

Gabriele Volpato

Freerk Wiersum

Peter Wilkin

Lixin Yang

Kowiyou Yessoufou

Scott Zona

The Society for Economic Botany extends its personal thanks to the women and men who agreed to peer reviewer manuscript submissions during 2015. It's a tough and often thankless job, and we sincerely appreciate your efforts. The number in parentheses indicates multiple reviews. We apologize for any names we may have missed. We also give a special thanks to our Associate Editors, who do most of the real work of the journal, and who are frequently called upon to review additional manuscripts. 